Med Exp Salud Publica. 2016;33(2):241-7. doi: 10.17843/rpmesp.2016.332.2170.

3. Santiago-Ullero B, Valer-Villanueva S, Urrunaga-Pastor D, BenitezZapata VA. Estrés percibido e intención de migrar al interior del país en Médicos y Enfermeros que residen en Lima: un análisis exploratorio de la Encuesta Nacional de Satisfacción de Usuarios en Salud (ENSUSALUD), 2015. Rev Peru Med Exp Salud Pública. 2017;34(3):404-13. doi: 10.17843/rpmesp.2017.343.2822.

4. Sánchez-Meca J, Alacid-de-Pascual I, López-Pina JA, Cruz Sánchez-Jiménez J. Meta-análisis de generalización de la fiabilidad del inventario de obsesiones de Leyton versión para niños autoaplicada. Rev Esp Salud Publica. 2016;90:e1-e14.

5. Loera B, Converso D, Viotti S. Evaluating the psychometric properties of the Maslach Burnout Inventory-Human Services Suvery (MBI-HSS) among Italian nurses: how many factors must a researcher consider? PLoS One. 2014;9(12):e114987. doi: 10.1371/journal.pone.0114987.

6. Olivares-Faúndez VE, Mena-Miranda L, Jélvez-Wilke C, MacíaSepúlveda F. Validez factorial del Maslach Burnout Inventory Service (MBI-HSS) en profesionales chilenos. Univ Psychol. 2014;13(1):145-59. doi: 10.11144/Javeriana.UPSY13-1.vfmb.

Correspondencia: César Merino Soto

Dirección: Calle Vieja 322, interior 116, Condominio Las Bugambilias

2, CP: Cuernavaca, Morelos, México.

Teléfono: (52) 7773644210

Correoelectrónico:sikayax@yahoo.com.ar

\section{INCENTIVOS POR PUBLICACIÓN CIENTIIFICA EN UNIVERSIDADES PERUANAS QUE CUENTAN CON ESCUELAS DE MEDICINA, 2017}

\section{INCENTIVES FOR SCIENTIFIC PUBLICATION IN PERUVIAN UNIVERSITIES THAT HAVE MEDICAL SCHOOLS, 2017}

Wendy Nieto-Gutierrez ${ }^{1, a}$, José Ernesto Fernández-Chinguel ${ }^{1, a}$, Alvaro Taype-Rondan', , Josmel Pacheco-Mendoza ${ }^{2, c}$, Percy Mayta-Tristán ${ }^{3, d}$

Sr. Editor. Las universidades tienen entre sus principales funciones la generación de conocimiento, lo que se traduce, entre otras acciones, en publicaciones científicas en revistas

\footnotetext{
Facultad de Medicina Humana, Universidad de San Martín de Porres. Lima, Perú.

Universidad San Ignacio de Loyola. Lima, Perú.

Dirección General de Investigación, Desarrollo e Innovación, Universidad Científica del Sur. Lima, Perú

a Estudiante de Medicina; ${ }^{b}$ médico epidemiólogo; ${ }^{\mathrm{c}}$ veterinario, magister en gestión de la información y el conocimiento; ${ }^{d}$ médico salubrista Recibido: 21/12/2017 Aprobado: 17/01/2018 En línea: 03/05/2018
}

Citar como: Nieto-Gutierrez W, Fernández-Chinguel JE, Taype-Rondan A, Pacheco-Mendoza J, Mayta-Tristán P. Incentivos por publicación científica en universidades peruanas que cuentan con escuelas de medicina, 2017. Rev Peru Med Exp Salud Publica. 2018;35(2):354-6. doi: 10.17843/rpmesp.2018.352.3327. indizadas. Es por ello que los rankings de universidades suelen valorar el número de publicaciones realizadas por autores afiliados a su universidad de procedencia.

Esto ha generado que las universidades implementen políticas para incentivar el desarrollo de la investigación, siendo una de estas la bonificación económica a los autores por las publicaciones que realizan en revistas científicas, esta estrategia ha demostrado elevar el número de publicaciones científicas en algunas instituciones ${ }^{(1)}$.

En Perú, luego de la reforma implementada con la Ley Universitaria $\mathrm{N}^{\circ} 30220$ del año 2014 (https://goo.gl/dui4kM), algunas universidades han implementado incentivos para que sus docentes, trabajadores y estudiantes incrementen la producción científica de sus instituciones. Sin embargo, no se cuentan con datos al respecto, por lo cual se realizó un estudio con el objetivo de describir los incentivos por publicación científica en universidades peruanas que cuentan con escuelas de medicina.

Se realizó un estudio observacional, descriptivo de corte transversal, cuyas unidades de análisis fueron las 57 escuelas de medicina del Perú, registradas hasta el 2016 (considerando las distintas filiales). Durante noviembre de 2017 se buscó información acerca de los incentivos económicos y no económicos que brindan estas escuelas o sus universidades por publicar en revistas científicas, durante el año 2017. Para ello, se consultaron las siguientes fuentes para cada escuela de medicina: 1) páginas web oficiales de las escuelas de medicina y las universidades, 2) entrevistas directas con los directores de las escuelas de medicina o llamadas telefónicas a los centros de administrativos de cada escuela, y 3) búsquedas en Google con los nombres de cada una de las universidades y los términos: «incentivos por publicación», «reconocimientos por publicación» y «pago económico por publicación».

De las 57 escuelas de medicina evaluadas el 2017, solo ocho brindaron algún incentivo económico; las cuales corresponden a seis universidades (puesto que dos escuelas fueron filiales). De estas, solo la Universidad Nacional San Antonio Abad del Cusco (UNSAAC) es de presupuesto público. Solo dos, además, otorgaban incentivos no económicos (diplomas). Las características de los financiamientos, según universidades, se muestran en la Tabla 1.

En cinco de las seis universidades, el monto del incentivo económico dependía de la base de datos donde estaba indizada la revista en la que se realizó la publicación. Sólo dos universidades, la Universidad Científica del Sur (UCSUR) y la Universidad Peruana de Ciencias Aplicadas (UPC) brindaron el incentivo económico diferenciado, acorde al cuartil de la revista (según Scopus o Web of Science) en la que se publicó el artículo.

Tres de las seis universidades comenzaron a brindar incentivos económicos desde el 2017, otra desde el 2016 
Tabla 1. Incentivos por publicación de artículos científicos brindados por universidades peruanas con escuelas de medicina, 2017

\begin{tabular}{|c|c|c|c|c|c|c|}
\hline Características & UNSAAC & USMP & URP & UCSUR & UPAO & UPC \\
\hline Región & Cusco & $\begin{array}{c}\text { Lima y } \\
\text { Lambayeque * }\end{array}$ & Lima & Lima & $\begin{array}{l}\text { La Libertad y } \\
\text { Piura * }\end{array}$ & Lima \\
\hline Año de inicio & 2017 & 2017 & 2017 & 2016 & 2015 & 2013 \\
\hline \multicolumn{7}{|l|}{ Aplica a } \\
\hline Docentes & Sí & Sí & Sí & Sí & Sí & Sí \\
\hline Estudiantes & Sí & Sí & No & No & Sí & No \\
\hline Por concurso & Sí & Sí & No & No & Sí & Sí \\
\hline \multicolumn{7}{|l|}{ Bases de datos consideradas } \\
\hline Latindex & No & Sí & No & No & Sí & No \\
\hline SciELO & Sí & Sí & Sí & Sí & Sí & No \\
\hline Medline & Sí & Sí & Sí & Sí & Sí & No \\
\hline Scopus & Sí & Sí & Sí & Sí & Sí & Sí \\
\hline Web of Science (WoS) & Sí & Sí & Sí & Sí & Sí & Sí \\
\hline \multicolumn{7}{|l|}{ Diferencias en los incentivos por } \\
\hline Bases de datos & Sí & No & Sí & Sí & Sí & Sí \\
\hline Cuartil Scopus /WoS & No & No & No & Sí & No & Sí \\
\hline Idioma & Sí & No & Sí & No & No & No \\
\hline Por tipo de artículo & No & No & No & Sí & No & No \\
\hline $\begin{array}{l}\text { Rango de bonificación por artículo } \\
\text { publicado }\end{array}$ & $\begin{array}{l}2025 \text { a } \\
8100 \text { PER }\end{array}$ & $\begin{array}{l}2000 \mathrm{a} \\
5000 \text { PER }\end{array}$ & $\begin{array}{c}1000 \mathrm{a} \\
3000 \text { PER }\end{array}$ & $\begin{array}{c}500 \mathrm{a} \\
8000 \mathrm{PER}\end{array}$ & $\begin{array}{c}600 \mathrm{a} \\
3000 \text { PER }\end{array}$ & $\begin{array}{c}4000 \mathrm{a} \\
13000 \mathrm{PER}\end{array}$ \\
\hline Incentivos no económicos & No & Diplomas & No & No & Diplomas & No \\
\hline Fuentes de información & $\begin{array}{l}\text { https://goo.gl/ } \\
\text { ct5Hb8 }\end{array}$ & $\begin{array}{l}\text { https://goo.gl/ } \\
\text { YxN8dR }\end{array}$ & $\begin{array}{l}\text { https://goo.gl/ } \\
\text { WYfX5K }\end{array}$ & $\begin{array}{c}\text { https://goo.gl/ } \\
\text { wYifia }\end{array}$ & $\begin{array}{c}\text { No disponible } \\
\text { en web }\end{array}$ & $\begin{array}{c}\text { https://goo.gl/ } \\
\text { jzpfWp }\end{array}$ \\
\hline
\end{tabular}

* Cada una de estas universidades tiene dos filiales (dos escuelas de medicina) en diferentes regiones. 1 PER $=0,307$ USD

UNSAAC: Universidad Nacional San Antonio Abad del Cusco, USMP: Universidad de San Martin de Porres,

URP: Universidad Ricardo Palma, UCSUR: Universidad Científica del Sur, UPAO: Universidad Privada Antenor Orrego, UPC: Universidad Peruana de Ciencias Aplicadas

y otra desde el 2015; lo cual refleja una adopción reciente de esta estrategia. La UPC fue la única universidad que comenzó tempranamente con estos incentivos, desde el 2013. Esta universidad también ha sido la que ha presentado un mayor incremento de publicaciones, en Scopus, en los últimos años. Es probable que la estrategia de incentivos haya influenciado en dicho incremento, sumado a la implementación de la graduación por artículo científico y al cambio en la estrategia de la enseñanza en investigación ${ }^{(2)}$.

Adicionalmente, en Perú, todas las universidades públicas, y profesores de universidades privadas que laboren en hospitales e institutos públicos, pueden recibir incentivos por publicación a través de las convocatorias que realiza el Consejo Nacional de Ciencia, Tecnología e Innovación Tecnológica (CONCYTEC) (http://portal.concytec.gob.pe). En cuanto a las universidades con escuelas de medicina que, a nuestro conocimiento, hayan tenido en algún momento sistemas de incentivos por publicación, figuran la Universidad Nacional Mayor de San Marcos, la Universidad Peruana Cayetano Heredia y la Universidad Nacional José Faustino Sánchez Carrión. Otras universidades sin escuelas de medicina que tienen incentivos vigentes son la Pontificia Universidad Católica del Perú y la Universidad San Ignacio de Loyola.
Por otro lado, si bien los incentivos por publicación pueden incrementar la producción científica de las instituciones, algunos estudios han descrito que también pueden ocasionar un descenso de la calidad de los trabajos publicados ${ }^{(3)}$, esto podría suceder con mayor frecuencia en países en desarrollo ${ }^{(4)}$. Una forma de disminuir este efecto podría ser a través de la implementación de estándares de calidad para los incentivos (indización, factor de impacto y tipo de artículo). También se ha detectado que incrementan las faltas éticas en publicación como la manipulación de datos, el plagio, la publicación redundante y los problemas de autoría y filiación ${ }^{(5)}$, por lo que se deben implementar mecanismos de vigilancia y sanción, como el Comité de Integridad Científica, implementado recientemente en la UCSUR.

En conclusión, en el Perú solo seis universidades que tienen escuelas de medicina ofrecen incentivos para la publicación científica, de las cuales, solo dos otorgan esta bonificación considerando el cuartil de la revista y tres incluyen a estudiantes como receptores de estos beneficios. Resulta necesario estudiar el impacto de estos incentivos, no solo en la cantidad sino también en la calidad de las publicaciones, además de implementar mecanismos de vigilancia de posibles faltas a la integridad científica.

Contribuciones de autoría: PMT, JPM y ATR tuvieron la idea del estudio y lo diseñaron, ATR, WNG y JEFC recolectaron los datos y 
escribieron el borrador, PMT y ATR analizaron los datos, PMT y JPM revisaron críticamente el manuscrito. Todos los autores aprobaron la versión final y se hacen responsables del contenido del mismo.

Fuente de financiamiento: financiado por los autores.

Conflictos de interés: WNG, JEFC y ATR han estudiado en la Universidad de San Martín de Porres. PMT estudia y enseña en la Universidad Privada Antenor Orrego, ha laborado en la Universidad Peruana de Ciencias Aplicadas y actualmente trabaja en la Universidad Científica del Sur.

\section{REFERENCIAS BIBLIOGRÁFICAS}

1. Andersen LB, Pallesen T. «Not just for the money?» How financial incentives affect the number of publications at Danish research institutions. Int Public Manag J. 2008;11(1):28-47. doi: $10.1080 / 10967490801887889$.

2. Mayta-TristánP.Tesisen formatode artículocientífico:oportunidad para incrementar la producción científica universitaria. Acta Med Peru. 2016;33(2):95-98.

3. Opstrup N. When and why do university managers use publication incentive payments? J High Educ Pol Manag. 2017;39(5):524-39. doi: 10.1080/1360080X.2017.1354756.

4. Aboal D, Tacsir E. The impact of subsidies on researcher's productivity: Evidence from a developing country. Res Evaluat. 2017;26(4):269-83. doi: 10.1093/reseval/rvx031.

5. Edwards MA, Roy S. Academic research in the $21^{\text {st }}$ century: maintaining scientific integrity in a climate of perverse incentives and hypercompetition. Environ Eng Sci. 2017;34(1):51-61. doi: 10.1089/ees.2016.0223.

Correspondencia: Percy Mayta-Tristán

Dirección: Av. Brasil 2169, Dpto 802, Jesús María. Lima, Perú

Teléfono: (51) 987532133

Correoelectrónico:pmayta@cientifica.edu.pe

\section{PUBLICACIÓN DUPLICADA DE UN REPORTE DE CASO}

\section{DUPLICATE PUBLICATION OF A CASE REPORT}

\author{
Franco Romaní ${ }^{1}$, J. Jhonnel Alarco ${ }^{1}$, \\ Hugo Arroyo-Hernández ${ }^{1}$
}

Un reporte de caso es una descripción detallada de los síntomas, signos, resultados de estudios auxiliares, tratamiento y sus complicaciones, así como del seguimiento

\footnotetext{
Comité Editorial de Revista Peruana de Medicina Experimental y Salud Pública, Instituto Nacional de Salud, Lima, Perú

Recibido: 10/05/2018 Aprobado: 16/05/2018 En línea: 22/05/2018
}

Citar como: Romaní F, Alarco JJ, Arroyo-Hernández H. Publicación duplicada de un reporte de caso. Rev Peru Med Exp Salud Publica. 2018;35(2):356-8. doi: $10.17843 /$ rpmesp.2018.352.3664. de un paciente, que es relevante comunicar a la comunidad científica dado su interés médico, científico o educacional ${ }^{(1,2)}$. Existen condiciones que hacen que un caso clínico pueda ser publicado como reporte de caso. Las principales son que reconozca o describa una enfermedad rara o nueva, que presente efectos terapéuticos, eventos adversos o complicaciones inesperadas de tratamientos, o que describa una presentación inusual de una enfermedad común o poco frecuente. Estos últimos tienen especial importancia desde el punto de vista educacional, pues amplían el abanico diagnóstico de los lectores médicos ${ }^{(1)}$.

Los reportes de caso son cada vez más frecuentes y han ganado espacio en las revistas biomédicas, incluyendo aquellas de salud pública y epidemiología ${ }^{(3)}$. Con dicho incremento podrían incrementarse también las malas prácticas científicas. Un estudio en revistas especializadas en radiología encontró que, entre 1993 y 2013, el 5 \% de casos de duplicación correspondieron a reportes y series de caso ${ }^{(4)}$.

Recientemente, en el cuarto número del volumen 30 del 2017 de la Revista de la Sociedad Peruana de Medicina Interna se publicó una carta al editor titulada ¿Publicación duplicada?, en la cual el comité revisor de dicha revista manifiesta su sospecha de una publicación duplicada. Los reportes de caso involucrados se titulan «Adolescente con inestabilidad lumbosacra por presencia de quiste hidatídico: reporte de caso» (Reporte $A){ }^{(5)} \mathrm{y}$ «Equinococosis vertebral»(Reporte $B)^{(6)}$. Dicha carta incluye la respuesta del editor, la cual a su vez presenta la respuesta de los autores. La presente comunicación analiza dicha respuesta utilizando las definiciones del Comité Internacional de Editores de Revistas Biomédicas (ICMJE) y los criterios sugeridos para identificar una publicación duplicada ${ }^{(7)}$.

EI ICMJE define publicación duplicada como la publicación de un manuscrito que se superpone sustancialmente con uno ya publicado, sin la debida referencia a la publicación anterior (https://goo.gl/bkxTTK). Esto puede suceder cuando los autores envían un manuscrito en forma simultánea a más de una revista, lo cual presumiblemente ha ocurrido en este caso. Los criterios para definir una publicación duplicada son el tamaño de muestral similar, metodología idéntica o muy parecida, resultados similares, al menos un autor común en ambas publicaciones, no se aporta información nueva o ésta es muy poco relevante ${ }^{(7)}$.

De la respuesta brindada por los autores y del análisis realizado a ambos reportes (Tabla 1), se concluye que se trata del mismo paciente y por ende del mismo caso clínico. Por otro lado, los autores manifiestan que su publicación no es duplicada por contar con una redacción y contenido diferente. Sin embargo, dicha aseveración no muestra una argumentación sólida respecto al no cumplimiento de los criterios de publicación duplicada. Se constata que existe un autor en común en ambas publicaciones (Mujica M). 